EPJ Web of Conferences 98, 04005 (2015)

DOI: $10.1051 /$ epjconf/ 20159804005

C Owned by the authors, published by EDP Sciences - SIF, 2015

\title{
Concentrated Photo Voltaics (CPV): a case study
}

\section{S. Centro}

Department of Physics and Astronomy, University of Padua

Associated to Centro "E. Fermi" - Rome, Italy

\begin{abstract}
Summary. - Concentrated PhotoVoltaic (CPV), among green energy solutions, nowadays has the ambition to reach grid-parity without subside. CPV substitutes large areas of expensive semiconductor solar cells, with concentrating optics made of cheap materials. Moreover the cells that are suitable for CPV exhibits an unprecedented efficiency and their technology is progressing every year. A case study project, TwinFocus ${ }^{\circledR}$, will be presented in detail.
\end{abstract}

\section{1. - Introduction}

CPV is conceptually simple. We need solar cells that stand concentration (not silicon), some optics to concentrate and a sun tracker. In principle concentration allows for reducing the semiconductor surface so reducing costs. Another benefit with CPV is the use of multiple-junction cells that have a higher efficiency compared with all other available cells so far. Moreover the multiple-junction cells have a lower efficiency dependence per temperature variation: we go from the $-0.4 \% / \mathrm{K}$ for silicon, to $-0.04 \% / \mathrm{K}$ for triple junction cells. A great advantage considering that usually concentration factors span from 400 to $1000 \times$. High concentrations mean critical thermal budget hence a low dependence of efficiency per temperature degree is extremely important.

This is an Open Access article distributed under the terms of the Creative Commons Attribution License 4.0, which permits unrestricted use, distribution, and reproduction in any medium, provided the original work is properly cited. 


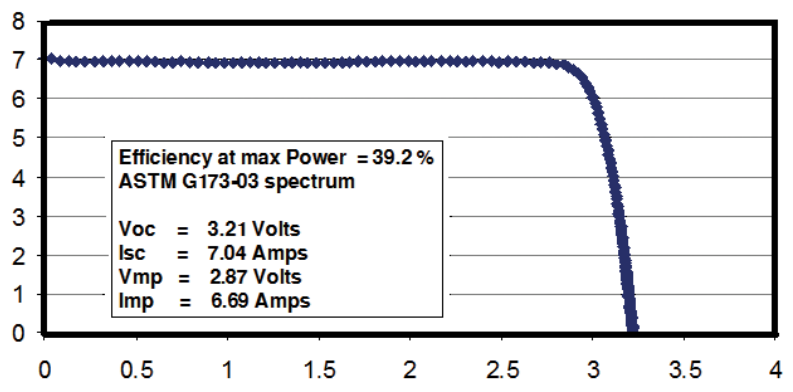

Fig. 1. $-I-V$ characteristic of a millimeter square $3 \mathrm{~J}$ cell showing the current characteristic from the open circuit point, $I_{O C}$, versus the cell voltage until the short circuit voltage, $V_{S C}$, where the 3 junctions are directly biased.

Silicon power plants frequently spoil large land areas, in principle higher efficiencies could save land or even the necessary use of tracker could permit dual land usage, provided the pole of the tracker is sufficiently high.

The saved surface of semiconductor may be substituted by cheap materials for implementation of the optics (see later). The challenge is to realize a system economically sustainable.

\section{2. - Triple-junction solar cells}

The triple-junction (3J) cells used in the project described afterword, are made of a sandwich of three junctions one on top of the other. On a metallized germanium substrate the first germanium junction is realized. This one has light sensitivity in the range $900-1800 \mathrm{~nm}$. On top of this junction another InGaAs junction is grown with a light sensitivity in the range $600-900 \mathrm{~nm}$.

Eventually a third junction InGaP is grown with sensitivity in the range 300-700 nm. It should be remembered that silicon has a sensitivity range from 200 to $1100 \mathrm{~nm}$. On top of the external layer, an aluminum electrode, made of very thin fingers, is deposited (cathode). The fingers are connected to two tiny busses, on the sides of the cell, and allow the sunlight to illuminate the cell while collecting the electrons generated by incident photons. There is a trade-off between finger thickness, spacing and profile to reach the minimum shading with the maximum electrons collection. The anode of the cell is obtained on the bottom metallized germanium substrate. The interconnection of the cells requires mastering some techniques typical of microelectronics: surface soldering of the anode on a copper support, that is one of the connection, and wire-bonding of the cathode busses on the other copper connection.

The $I-V$ characteristic of a 3J cell is given in fig. 1 . The cell behaves as a current generator, whose current, $I_{O C}$, depends upon irradiation, until it reaches the voltage the 3 junctions become directly polarized, $V_{S C}$. At that point the current flows inside the cell through the junctions. 


\section{LNES 2014}

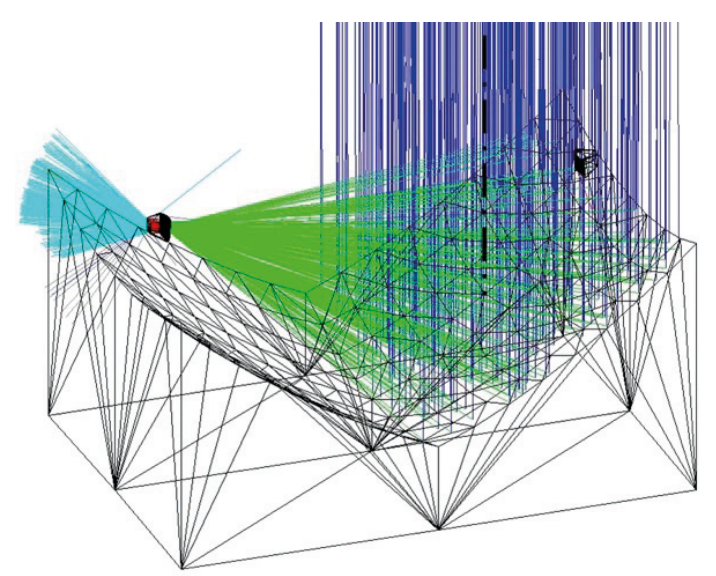

Fig. 2. - Ray tracing of light on a half TwinFocus ${ }^{\circledR}$ quasi-parabolic mirror surface. The incident light, blue rays, is reflected, green rays, on a $3 \mathrm{~J}$ cell. The structure has a symmetrical unit in front.

It is important to notice that the three junctions, in the active region, behave as three current generators connected in series. Everybody knows that this should never be made in electronics. However it is the only way to operate cells in the real world. It is evident that all the three junctions should be irradiated with the proper intensity and wavelength, otherwise the most inefficient one will determine the overall performance in terms of delivered current.

\section{3. - The TwinFocus ${ }^{\circledR}$ project}

After funding of the CPV project through Centro "E. Fermi" with sizeable sums and mainly with junior grants and fellowship position, the project proponents founded an University spin-off, AtemEnergia srl, participated by the University. AtemEnergia succeeded in designing an innovative optics system for CPV.

The basic idea behind the project is to implement CPV with sound, mature, reliable and affordable technologies. The technology that is paramount for these characteristics is automotive, and in particular automotive lighting.

The second choice was to use reflectors rather than refractive element as primary optics. A cheap lens has large chromatic aberration, that spoil the 3J structure where the junctions must be evenly irradiated, while mirrors do not have chromatic aberration.

Another choice was to avoid shading of the reflector as much as possible with secondary optics, that must be as small as possible. This choice implies the design of very demanding off-axis non-imaging optics. The outcome was a set of two quasi-parabolic adjacent mirrors that illuminate two opposite cells, almost vertical, as shown in fig. 2 . The name TwinFocus ${ }^{\circledR}$ comes from this characteristic original shape that allows for high concentration factors while maintaining unprecedented element low thickness. 


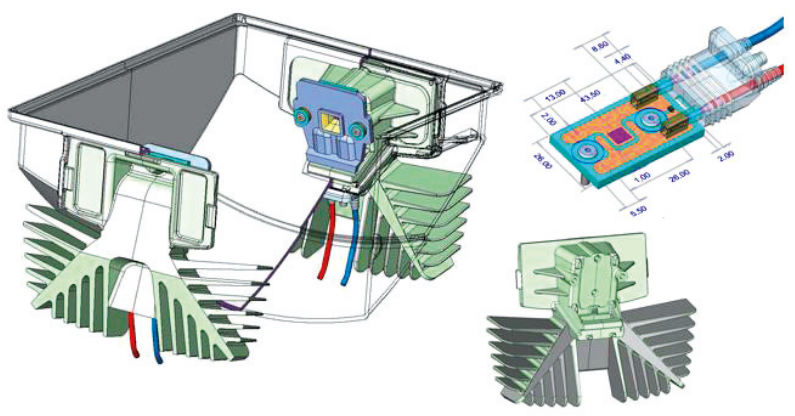

Fig. 3. - Rendering of a TwinFocus ${ }^{\circledR}$ unit, with details of cell support and heat sink.

In the first series of TwinFocus ${ }^{\circledR}$ modules 3J cells, $5 \times 5 \mathrm{~mm}^{2}$, have been used with concentration factor of $580 \times$. The mirrors area is $22 \times 18 \mathrm{~cm}^{2}$ with a depth of only $14 \mathrm{~cm}$.

One relevant shareholder of AtemEnergia, Unitek srl, set up a network of industries that decided to implement the TwinFocus ${ }^{\circledR}$ project. Unitek is a company leader in mold
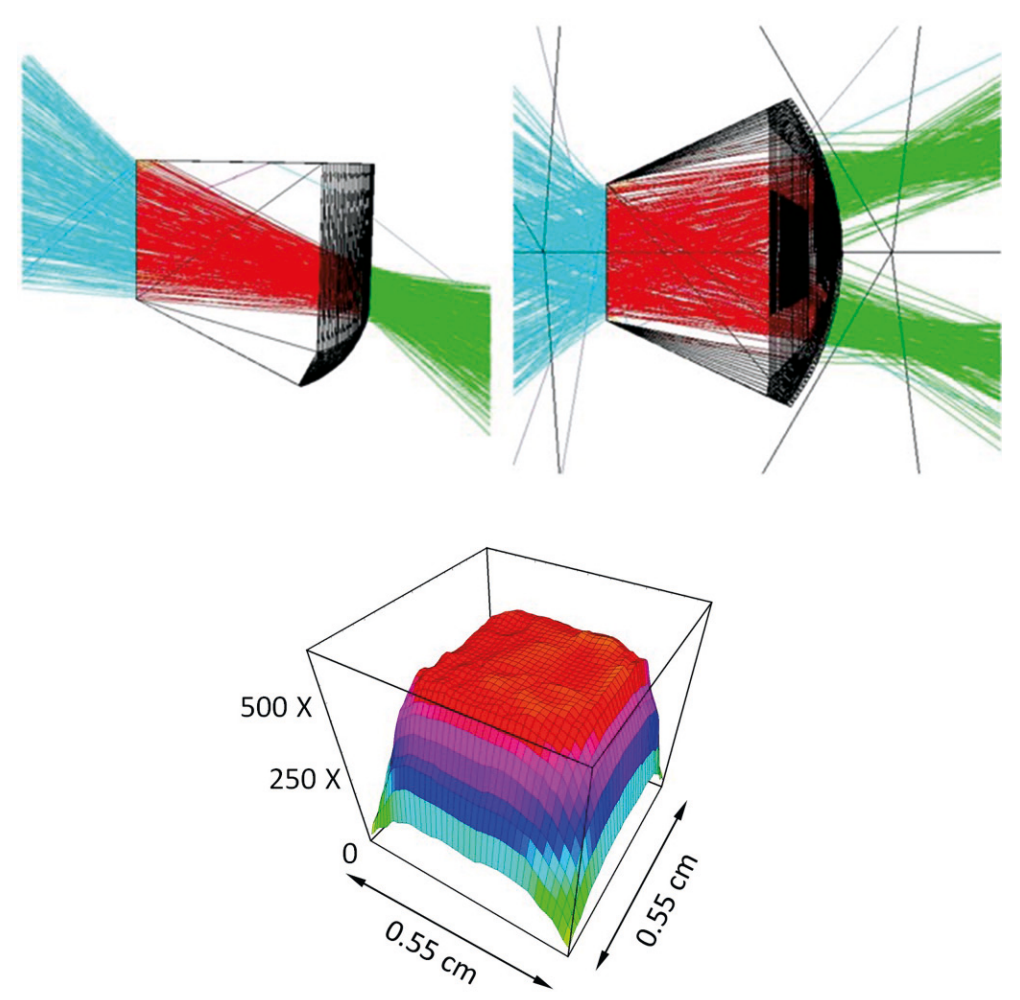

Fig. 4. - Lateral and top ray tracing are shown in the top part, while illumination profile is in the bottom part. 


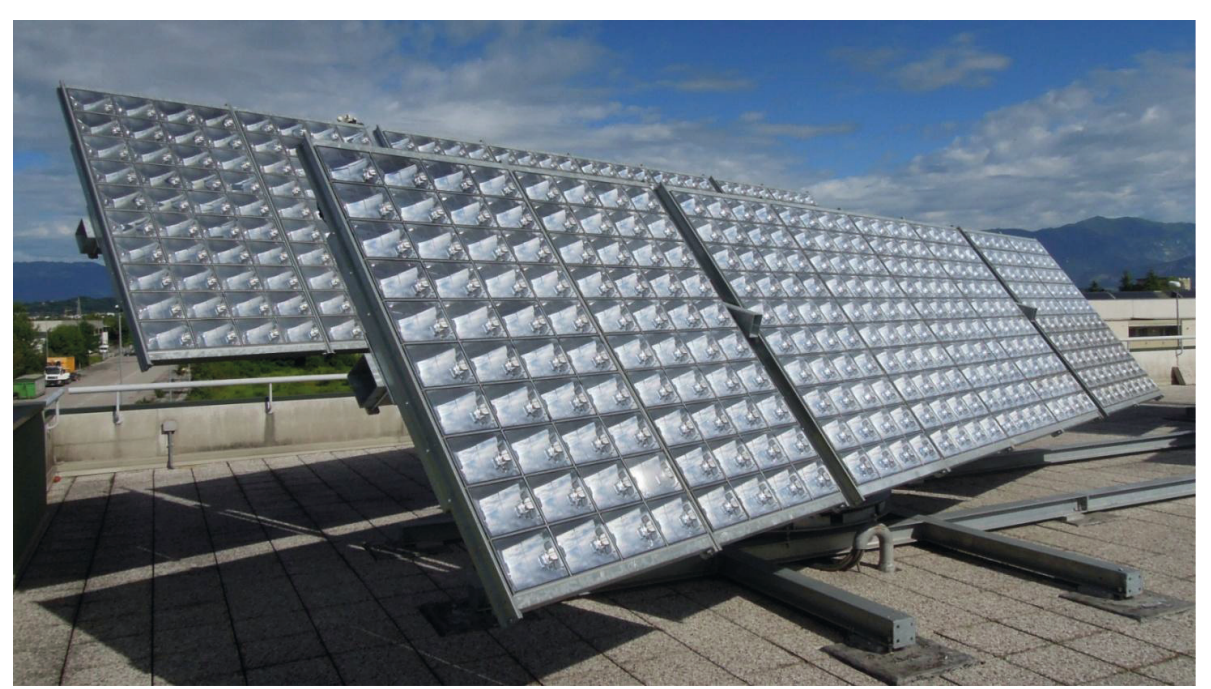

Fig. 5. - A ring tracker on the roof of the Unitek production site.

design and construction, mainly for automobile head-lights. The network of industries was composed by PiazzaRosa, for mold optical polishing, and Unica for polycarbonate injection.

The TwinFocus ${ }^{\circledR}$ mirrors are realized in injected polycarbonate, with aluminum coating, with aluminum heat sink for cells, glass and copper. All materials are recycled or recyclable, certainly a plus respect to standard silicon solar systems.

A rendering of the basic units is shown in fig. 3, where details of cell support, heat sink and overall unit are visible.

The detail of the off axis optical design, that used a light guide, made of molded quartz, as secondary optics is shown in fig. 4.

Four prototypes were realized and installed in the industries premises. They demonstrated the viability of the adopted solutions and they produce energy used by the companies. The modules are mounted on ring trackers for an installed power of about $5 \mathrm{kWp}$. One of them is shown in fig. 5 .

The CPV is suitable for areas with high Direct Normal Irradiation (DNI), but even in North East of Italy, where all the participants are based, we obtained in days with high DNI exceptional results. With DNI in the order of $880 \mathrm{~W} / \mathrm{m}^{2}$ we measured for a single TwinFocus ${ }^{\circledR}$ unit $7,96 \mathrm{~W}$ power with Fill Factor (FF) of $82 \%$ and an efficiency in excess of $25 \%$. These data are summarized in figs. 6 and 7 where also a real TwinFocus ${ }^{\circledR}$ unit is shown together with measured parameters and characteristic.

\section{4. - The TwinFocus ${ }^{\circledR}$ evolution}

Although the reported performances are quite satisfactory, a critical examination of the units immediately suggests a few fundamental improvements. The dimensions of the 


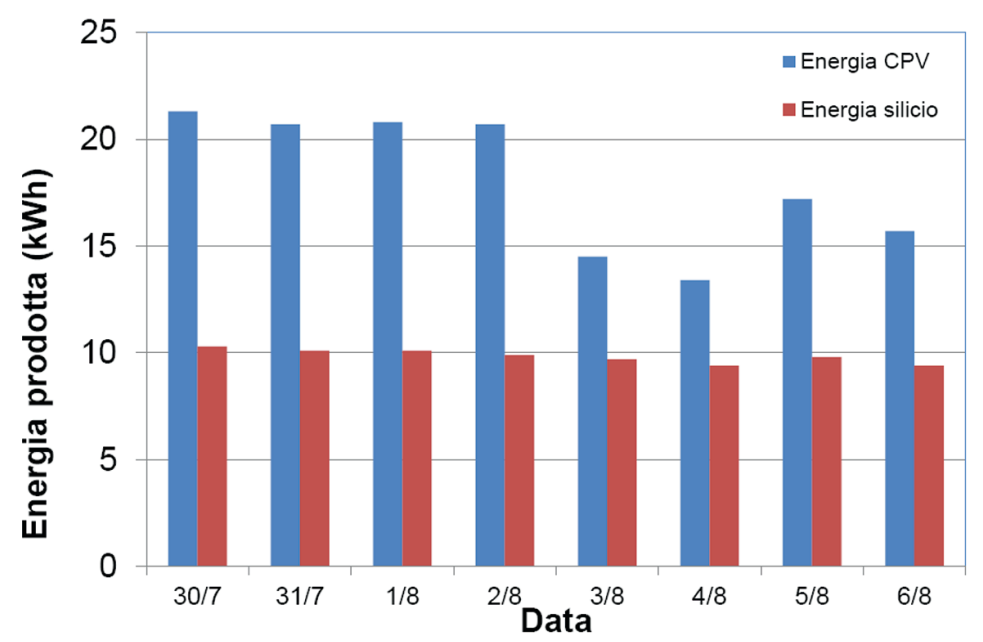

Fig. 6. - Comparison of energy produced in a few days in 2012, with same area of CPV and PV.

unit, that is the building block of the system as shown in fig. 7, is too small and requires a matrix structure for the mechanical support. Moreover four cables for each units are expensive and make the wiring cumbersome. Eventually the cell position protrudes from the lateral side and cast shadow on the mirror.

The TwinFocus ${ }^{\circledR}$ new design solves all the listed limitations. The mechanical structure holds the cells and performs heat sinking at the same time. The cells are kept vertical and the secondary optics (molded quartz) does not cast any shadow. The concentration factor is then increased and the wiring is simplified. The basic module is shown in fig. 8 where the rendering of three mirrors is shown. Two bars of extruded aluminum hold the

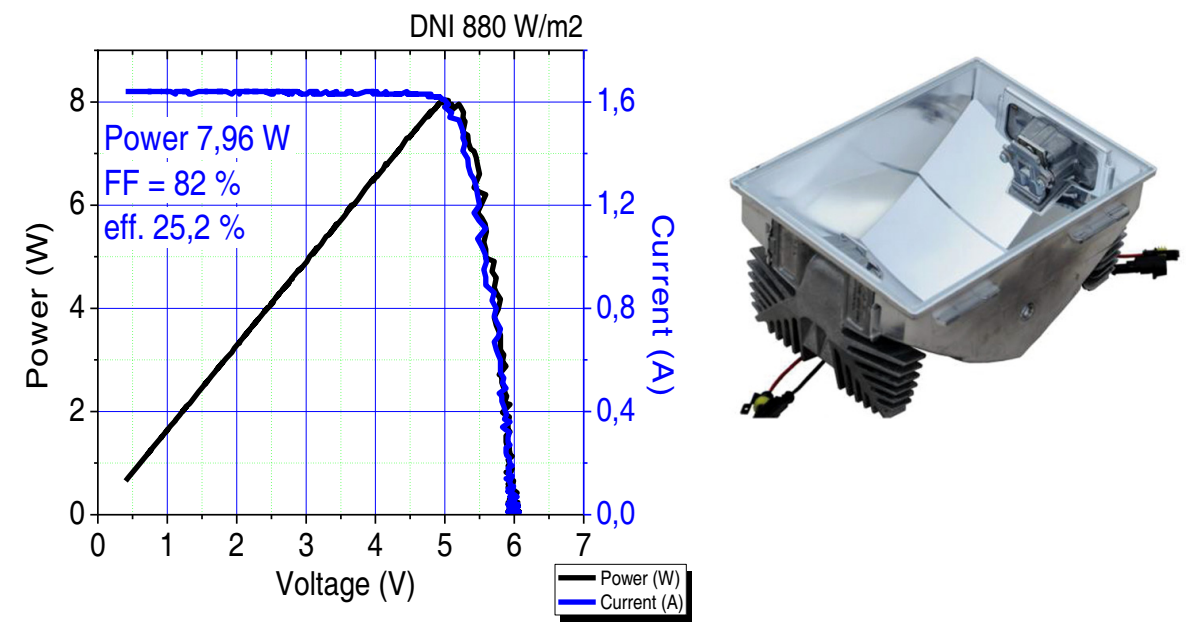

Fig. 7. - A TwinFocus ${ }^{\circledR}$ unit at the right with measured parameters in the graph at the left. 


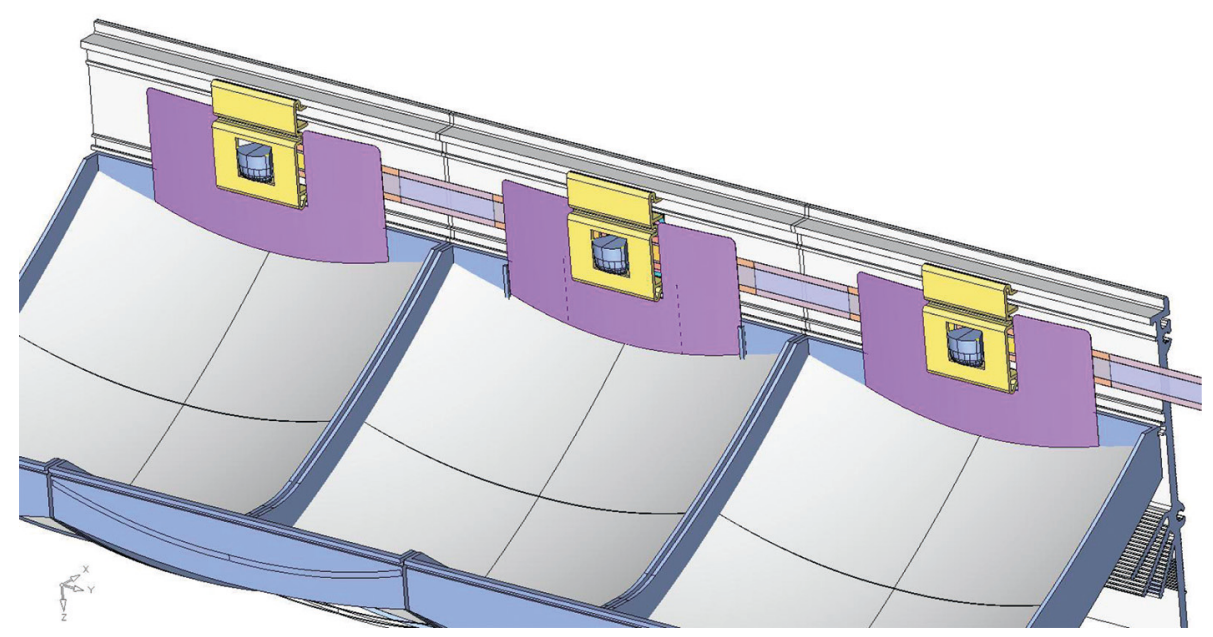

Fig. 8. - In the new TwinFocus ${ }^{\circledR}$ design, one module is made of a series of 12 mirrors kept in place by two extruded aluminum profiles that provide also heat sinking. The $243 \mathrm{~J}$ cells are mounted with direct dovetail joint without any screw.

cells and the mirrors at the same time. The proprietary design allows for insertion of all the pieces with dovetail technique without any screw. The full TwinFocus ${ }^{\circledR}$ module now, according to the structure of fig. 8, is made of 12 mirrors held together by the extruded aluminum with two caps at the two edges. Only the two caps require two times 4 screws to be held in place.

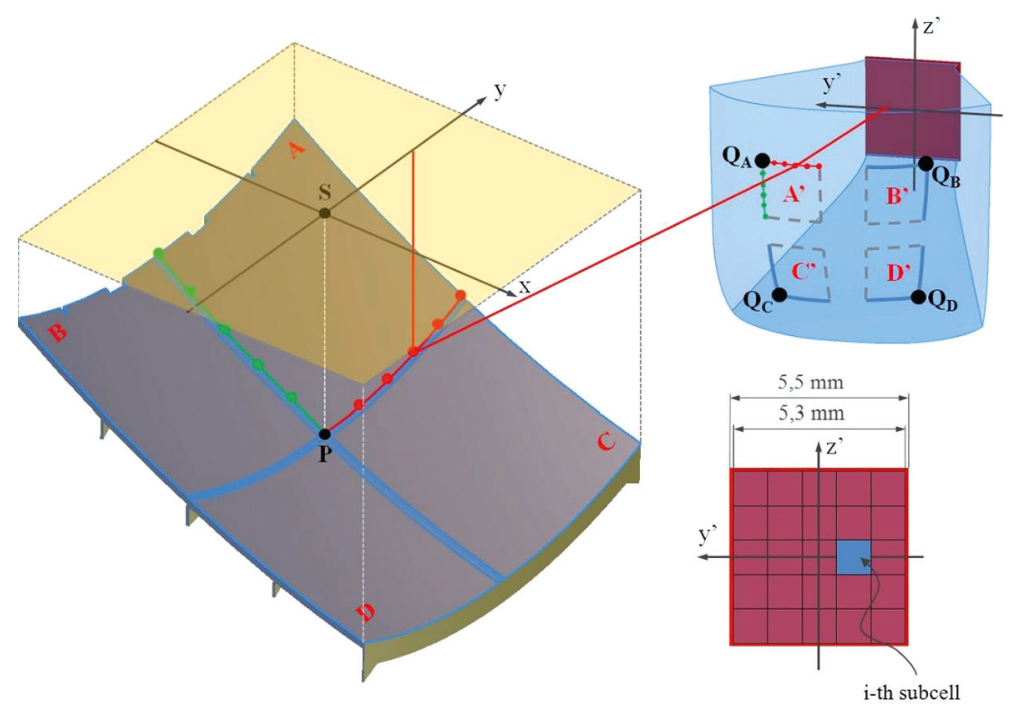

Fig. 9. - The new optical design collects more rays and reaches a more even cell illumination. 


\section{EPJ Web of Conferences}
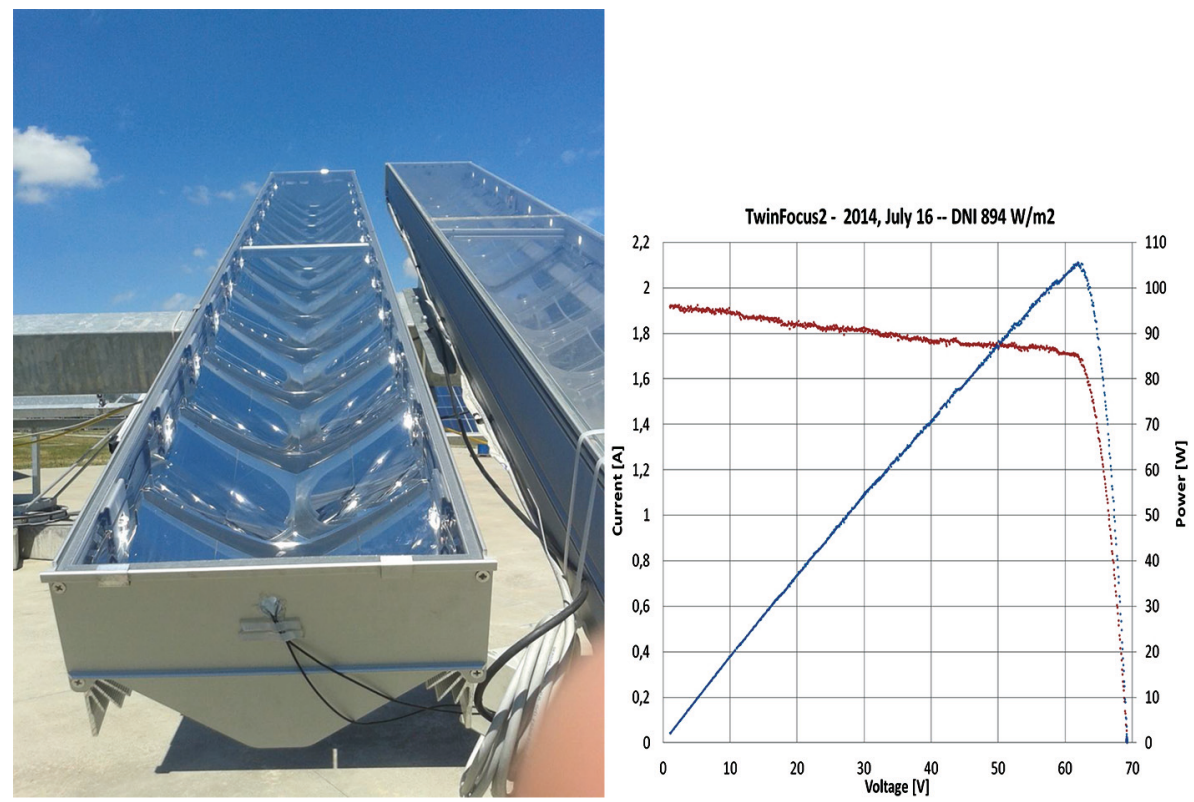

Fig. 10. - A module under test and the measured current and power versus voltage on field.

The structure shown in fig. 8 simplifies enormously the electrical interconnections. The full module is $2.3 \mathrm{~m}$ long, $29 \mathrm{~cm}$ wide and $16 \mathrm{~cm}$ thick.

Concentration is in the excess of 760 suns.

Also the off-axis optical design, shown in fig. 9, has been improved to obtain a more even cell illumination and larger tolerance in case of non-perfect alignment.

The new modules are now under test and their production will start soon for a pilot installation in central Italy.

So far, with proprietary active load, the fill factor has been evaluated and in fig. 10 power graph and module assembled are shown. The measured efficiency is $26 \%$.

The CPV project has been possible only because of the strong support of Centro "E. Fermi" that has acknowledged the CPV activity as one of the projects recognized in the framework of "Energy". The author is indebted toward Piergiorgio Antonini, Fermi Junior Grant, for the project management, Alessandro Saccà, Fermi post-doc fellow, for non-imaging optical design, and Stelvio Golfetto for field measurements, thermal simulation and design. A special thank to Guang Meng that made simulation and design of the active load for unit testing. Eventually the strong investment effort made by industrial partners cited in sect. 3 must be recognized. The industrial network, with the plus of the collaboration with Centro "E. Fermi" as research support, had access to significant funding from Assessorato all'Economia e Sviluppo, Ricerca e Innovazione of Veneto Regional Government, for the realization of the four demonstrators. 